\title{
Positive properties of the Green function for two-term fractional differential equations and its application
}

\author{
Yongqing Wang ${ }^{a, b, *}$, Lishan Liu \\ a School of Statistics, Qufu Normal University, Qufu 273165, Shandong, P. R. China. \\ ${ }^{b}$ School of Mathematical Sciences, Qufu Normal University, Qufu 273165, Shandong, P. R. China. \\ ${ }^{c}$ Department of Mathematics and Statistics, Curtin University, Perth, WA6845, Australia.
}

Communicated by X.-J. Yang

\begin{abstract} equation

In this paper, we study the positive properties of the Green function for the following two-term fractional differential

$$
\left\{\begin{array}{l}
-D_{0+}^{\alpha} u(t)+b u(t)=f(t, u(t)), \quad 0<t<1, \\
u(0)=0, \quad u(1)=0,
\end{array}\right.
$$

where $1<\alpha<2, \mathrm{~b}>0, \mathrm{D}_{0+}^{\alpha}$ is the standard Riemann-Liouville derivative. As an application, the existence and uniqueness of positive solution are obtained under the singular conditions. Moreover, an iterative scheme is established to approximate the unique positive solution. (C)2017 All rights reserved.
\end{abstract}

Keywords: Multi-term fractional differential equation, Green function, iterative solution, boundary value problems. 2010 MSC: 34A08, 34B27.

\section{Introduction}

It has been proved that fractional-order models are more accurate than integer-order models as fractional order models allow more degrees of freedom. For example, fractional derivatives in the sense of Caputo type can be used to describe the anomalous behaviors of diffusive phenomena [25-30]. Fractional differential equations (FDE) serve as an excellent instrument for the description of memory and hereditary properties of various materials and processes. Recently, much attention has been paid to the study of boundary value problems (BVP) of fractional differential equation, such as the singular BVP $[17,21,33,34]$, nonlocal BVP [2, 5, 20, 24], semipositone BVP [19, 22, 23] and resonant BVP [3, 4, 18, 32].

Multi-term fractional differential equations have been used to model various types of visco-elastic damping [1, 14]. The proposed model equations are almost always linear. Many authors focused on equations of the linear form:

$$
\left[D^{\alpha_{N}}+b_{N-1} D^{\alpha_{N-1}}+\cdots+b_{1} D^{\alpha_{1}}+b_{0} D^{0}\right] y(t)=g(t),
$$

\footnotetext{
*Corresponding author

Email addresses: wyqing9801@163.com (Yongqing Wang), 1ls@mail.qfnu.edu.cn (Lishan Liu)
} 
where $b_{i} \in \mathbb{R}(i=0,1, \cdots, N-1)$, equipped with initial conditions [6, 8-10]. In [9], the authors investigated the Endolymph equation:

$$
D^{2} x(t)+a_{1} D x(t)+a_{2} D^{\frac{1}{2}} x(t)+a_{3} x(t)=-g(t),
$$

which can be used to describe model for the response of the semicircular canals to the angular acceleration. By using the Laplace transformation, an exact solution was obtained for the equation of motion. In [8], by using the method of separation of variables, the authors investigated the following multi-term of fractional diffusion-wave equation along with the homogeneous/non-homogeneous boundary conditions:

$$
P(D) u(x, t)=k \frac{\partial^{2} u(x, t)}{\partial x^{2}}+q(t), \quad 0<x<\pi, \quad t>0,
$$

where

$$
\mathrm{P}(\mathrm{D})=\mathrm{D}_{\mathrm{t}}^{\mu}-\sum_{i=1}^{\mathrm{r}-1} \lambda_{i} \mathrm{D}_{\mathrm{t}}^{\mu_{\mathrm{i}}}, \quad 0<\mu_{\mathrm{r}-1}<\mu_{\mathrm{r}-2}<\cdots<\mu_{1}<\mu \leqslant 2 .
$$

It should be noted that the solution is not necessarily non-negative, and hence does not represent anomalous diffusion of any kind.

Since only positive solutions are meaningful in most practical problems, some work has been done to study the existence of positive solutions for fractional boundary value problems (FBVP) by using the techniques of nonlinear analysis such as fixed point theorems, Leray-Schauder theory, etc. We refer to the references $[7,11,16,31,35]$. It is well-known that the cone plays a very important role in seeking positive solutions of FBVP. Moreover, the cone is usually derived from the positive properties of the Green function. In [12], the authors discussed some positive properties of the Green function for Dirichlet-type FBVP, and obtained the existence of positive solutions by using the Krasnosel'skii fixed-point theorem. In [24], the authors investigated the following fractional differential equation:

$$
\left\{\begin{array}{l}
-D_{0+}^{\alpha} u(t)=f(t, u(t))+e(t), 0<t<1, \\
u(0)=0, D_{0+}^{\beta} u(1)=a D_{0+}^{\beta} u(\xi),
\end{array}\right.
$$

where $1<\alpha \leqslant 2,0<\beta \leqslant \alpha-1,0<\xi<1,0 \leqslant a \leqslant 1$ and $a \xi^{\alpha-\beta-2} \leqslant 1-\beta$. The authors obtained some properties of the Green function. But they failed to obtain the positive properties similar to that of [12] in form, and given an open problem about positive properties of the Green function, that is, [24, Remark 2.1]. Wang et al. [21,22] established some new positive properties of the corresponding Green function, and solved the open problem of [24]. As application, the existence of positive solutions were obtained for a class of fractional m-point BVPs.

Inspired by the above works, in this paper, we aim to deduce some positive properties of the Green function for the following Dirichlet-type FBVP

$$
\left\{\begin{array}{l}
-D_{0+}^{\alpha} u(t)+b u(t)=f(t, u(t)), 0<t<1, \\
u(0)=0, u(1)=0,
\end{array}\right.
$$

where $1<\alpha<2, b>0, D_{0+}^{\alpha}$ is the standard Riemann-Liouville derivative. The paper is organized as follows. In Section 2, we present some preliminaries and lemmas that will be used to prove our main results. In Section 3, we establish some positive properties of the Green function. In Section 4, we discuss the existence and uniqueness of positive solution for FBVP (1.1) under the singular conditions, that is, $f(t, x)$ may be singular at $t=0,1$, and $x=0$. Moreover, we establish an iterative scheme to approximate the unique positive solution.

\section{Basic definitions and preliminaries}

Definition 2.1. The fractional integral of order $\alpha>0$ of a function $u:(0,+\infty) \rightarrow R$ is given by

$$
I_{0+}^{\alpha} u(t)=\frac{1}{\Gamma(\alpha)} \int_{0}^{t}(t-s)^{\alpha-1} u(s) d s,
$$


provided that the right-hand side is point-wise defined on $(0,+\infty)$.

Definition 2.2. The Riemann-Liouville fractional derivative of order $\alpha>0$ of a function $u:(0,+\infty) \rightarrow R$ is given by

$$
D_{0+}^{\alpha} u(t)=\frac{1}{\Gamma(n-\alpha)}\left(\frac{d}{d t}\right)^{n} \int_{0}^{t}(t-s)^{n-\alpha-1} u(s) d s,
$$

where $n=[\alpha]+1,[\alpha]$ denotes the integer part of number $\alpha$, provided that the right-hand side is pointwise defined on $(0,+\infty)$.

Denote

$$
g(t)=\frac{\alpha-2}{\Gamma(\alpha-1)}+\sum_{k=1}^{+\infty} \frac{t^{k}}{\Gamma((k+1) \alpha-2)}
$$

It is easy to check that

$$
g(0)=\frac{\alpha-2}{\Gamma(\alpha-1)}<0, \quad g^{\prime}(t)>0, \quad \text { on } \quad[0,+\infty)
$$

and

$$
\lim _{t \rightarrow+\infty} g(t)=+\infty
$$

Therefore, there exists a unique $b^{*}>0$ such that

$$
g\left(b^{*}\right)=0 .
$$

Throughout this paper, we always suppose that the parameter $b$ in (1.1) satisfies $\left(\mathrm{H}_{1}\right) \quad \mathrm{b} \in\left(0, \mathrm{~b}^{*}\right]$.

Denote

$$
G(t)=t^{\alpha-1} E_{\alpha, \alpha}\left(b t^{\alpha}\right)
$$

where

$$
\mathrm{E}_{\alpha, \alpha}(\mathrm{x})=\sum_{\mathrm{k}=0}^{+\infty} \frac{\mathrm{x}^{\mathrm{k}}}{\Gamma((\mathrm{k}+1) \alpha)}
$$

is the Mittag-Leffler function [13, 15]. Set

$$
K(t, s)=\frac{1}{G(1)}\left\{\begin{array}{l}
G(t) G(1-s), \quad 0 \leqslant t \leqslant s \leqslant 1, \\
G(t) G(1-s)-G(t-s) G(1), \quad 0 \leqslant s \leqslant t \leqslant 1 .
\end{array}\right.
$$

Lemma 2.3. Suppose that $\left(\mathrm{H}_{1}\right)$ holds, and $\mathrm{y} \in \mathrm{L}[0,1]$. Then the problem

$$
\left\{\begin{array}{l}
-D_{0+}^{\alpha} u(t)+b u(t)=y(t), \quad 0<t<1 \\
u(0)=0, u(1)=0
\end{array}\right.
$$

has a unique solution

$$
u(t)=\int_{0}^{1} K(t, s) y(s) d s .
$$

Proof. As argued in $[13,15]$, the general solution of (2.2) can be expressed by

$$
u(t)=-\int_{0}^{t} G(t-s) y(s) d s+c_{1} G(t)+c_{2} G^{\prime}(t) .
$$

Since $u(0)=0$, we have $c_{2}=0$. 
On the other hand,

$$
u(1)=-\int_{0}^{1} G(1-s) y(s) d s+c_{1} G(1)
$$

So,

$$
c_{1}=\frac{\int_{0}^{1} G(1-s) y(s) d s}{G(1)} .
$$

Therefore, the solution of (2.2) is

$$
\begin{aligned}
u(t) & =-\int_{0}^{t} G(t-s) y(s) d s+\frac{\int_{0}^{1} G(1-s) y(s) d s}{G(1)} G(t) \\
& =\frac{\int_{0}^{1} G(t) G(1-s) y(s) d s-\int_{0}^{t} G(1) G(t-s) y(s) d s}{G(1)} \\
& =\int_{0}^{1} K(t, s) y(s) d s .
\end{aligned}
$$

\section{Main results}

Theorem 3.1. Suppose that $\left(\mathrm{H}_{1}\right)$ holds. Then the function $\mathrm{K}(\mathrm{t}, \mathrm{s})$ has the following properties:

(1) $\mathrm{K}(\mathrm{t}, \mathrm{s})>0, \quad \forall \mathrm{t}, \mathrm{s} \in(0,1)$;

(2) $\mathrm{K}(\mathrm{t}, \mathrm{s})=\mathrm{K}(1-\mathrm{s}, 1-\mathrm{t}), \quad \forall \mathrm{t}, \mathrm{s} \in[0,1]$;

(3) $\mathrm{K}(\mathrm{t}, \mathrm{s}) \leqslant \mathrm{G}(1) \mathrm{s}(1-\mathrm{s})^{\alpha-1} \mathrm{t}^{\alpha-2}, \quad \forall \mathrm{t}, \mathrm{s} \in[0,1]$;

(4) $\mathrm{K}(\mathrm{t}, \mathrm{s}) \geqslant M s(1-s)^{\alpha-1}(1-\mathrm{t}) \mathrm{t}^{\alpha-1}, \forall \mathrm{t}, \mathrm{s} \in[0,1]$, where

$$
M=\min \left\{\frac{1}{G(1)[\Gamma(\alpha)]^{2}}, G(1)(\alpha-1)^{2}\right\}
$$

Proof. We only need to prove that (3) and (4) hold.

By (2.1), we have

$$
\begin{gathered}
\frac{t^{\alpha-1}}{\Gamma(\alpha)} \leqslant G(t)=t^{\alpha-1} \sum_{k=0}^{+\infty} \frac{b^{k} t^{\alpha k}}{\Gamma((k+1) \alpha)} \leqslant t^{\alpha-1} G(1), \quad t \in[0,1] \\
G^{\prime}(t)=\sum_{k=0}^{+\infty} \frac{b^{k} t^{(k+1) \alpha-2}}{\Gamma((k+1) \alpha-1)}>0, \quad t \in(0,1]
\end{gathered}
$$

and

$$
\begin{aligned}
G^{\prime \prime}(t) & =t^{\alpha-3}\left[\frac{\alpha-2}{\Gamma(\alpha-1)}+\sum_{k=1}^{+\infty} \frac{b^{k} t^{k \alpha}}{\Gamma((k+1) \alpha)-2}\right] \\
& =t^{\alpha-3} g\left(b t^{\alpha}\right)<t^{\alpha-3} g(b) \leqslant t^{\alpha-3} g\left(b^{*}\right)=0, \quad t \in(0,1),
\end{aligned}
$$

which implies that $G(t)$ is strictly increasing on $[0,1]$, and $G^{\prime}(t)$ is strictly decreasing on $(0,1]$. Moreover, it is easy to see that $G^{\prime \prime}(t)$ is strictly increasing on $(0,1]$.

(i) For $0<t \leqslant s<1$. By (3.1), we have

$$
\begin{aligned}
K(t, s) & =\frac{G(t) G(1-s)}{G(1)} \leqslant \frac{G(t) G(1-s)}{G(1)} \frac{1-t}{1-s} \\
& \leqslant G(1)(1-s)^{\alpha-1} t^{\alpha-1} \frac{1-t}{1-s} \\
& =G(1)(1-t) t^{\alpha-1}(1-s)^{\alpha-2},
\end{aligned}
$$


and

$$
\begin{aligned}
K(t, s) & =\frac{G(t) G(1-s)}{G(1)} \geqslant \frac{G(t) G(1-s)(1-t) s}{G(1)} \\
& \geqslant \frac{s(1-s)^{\alpha-1}(1-t) t^{\alpha-1}}{G(1)[\Gamma(\alpha)]^{2}} .
\end{aligned}
$$

(ii) For $0 \leqslant s<t \leqslant 1$. Following the monotonicity of $G(t), G^{\prime}(t)$ and $G^{\prime \prime}(t)$, we have

$$
\begin{aligned}
\frac{\partial}{\partial t} K(t, s) & =\frac{G^{\prime}(t) G(1-s)-G^{\prime}(t-s) G(1)}{G(1)} \\
& <\frac{G^{\prime}(t) G(1)-G^{\prime}(t-s) G(1)}{G(1)}<0,
\end{aligned}
$$

and

$$
\begin{aligned}
\frac{\partial^{2}}{\partial t^{2}} K(t, s) & =\frac{G^{\prime \prime}(t) G(1-s)-G^{\prime \prime}(t-s) G(1)}{G(1)} \\
& >\frac{G^{\prime \prime}(t) G(1)-G^{\prime \prime}(t-s) G(1)}{G(1)} \\
& =G^{\prime \prime}(t)-G^{\prime \prime}(t-s)>0,
\end{aligned}
$$

which implies $K(t, s) \geqslant K(1, s)=0$, and $\frac{\partial}{\partial t} K(t, s)$ is strictly increasing with respect to $t$ on $(s, 1]$. Therefore,

$$
\begin{aligned}
\frac{\partial}{\partial t}\left[\frac{K(t, s)}{1-t}\right] & =\frac{(1-t) \frac{\partial}{\partial t} K(t, s)+K(t, s)}{(1-t)^{2}} \\
& =\frac{\frac{\partial}{\partial t} K(t, s)-\frac{K(1, s)-K(t, s)}{(1-t)}}{(1-t)} \\
& =\frac{\frac{\partial}{\partial t} K(t, s)-\frac{\partial}{\partial t} K(\xi, s)}{(1-t)}
\end{aligned}
$$

where $t<\xi<1$. By the monotonicity of $\frac{\partial}{\partial t} K(t, s)$, we have $\frac{\partial}{\partial t}\left[\frac{K(t, s)}{1-t}\right] \leqslant 0$. Thus,

$$
\begin{aligned}
\frac{K(t, s)}{1-t} & \leqslant \frac{K(s, s)}{1-s}=\frac{G(1-s) G(s)}{G(1)(1-s)} \leqslant \frac{G(1-s) G(t)}{G(1)(1-s)} \\
& \leqslant \frac{G(1) t^{\alpha-1}(1-s)^{\alpha-1}}{1-s}=G(1) t^{\alpha-1}(1-s)^{\alpha-2},
\end{aligned}
$$

which implies that

$$
K(t, s) \leqslant G(1)(1-t) t^{\alpha-1}(1-s)^{\alpha-2}
$$

On the other hand, by the monotonicity of $G^{\prime}(t)$, we have

$$
\begin{aligned}
\frac{\partial}{\partial s} K(t, s) & =\frac{G^{\prime}(t-s) G(1)-G(t) G^{\prime}(1-s)}{G(1)} \\
& \geqslant \frac{[G(1)-G(t)] G^{\prime}(1-s)}{G(1)} .
\end{aligned}
$$

Integrate (3.5) with respect to s, we obtain

$$
\begin{aligned}
K(t, s) & \geqslant \int_{0}^{s} \frac{[G(1)-G(t)] G^{\prime}(1-\tau)}{G(1)} d \tau \\
& =\frac{[G(1)-G(t)][G(1)-G(1-s)]}{G(1)} .
\end{aligned}
$$


Since

$$
\frac{\mathrm{d}}{\mathrm{d} s}\left[(\alpha-1)(1-\mathrm{s})+\mathrm{s}^{\alpha-1}\right]=(\alpha-1)\left[\mathrm{s}^{\alpha-2}-1\right] \geqslant 0, \quad s \in(0,1]
$$

we have

$$
1-s^{\alpha-1} \geqslant(\alpha-1)(1-s)
$$

From (3.1), (3.6) and (3.7), we get

$$
\begin{aligned}
K(t, s) & \geqslant \frac{\left[G(1)-t^{\alpha-1} G(1)\right]\left[G(1)-(1-s)^{\alpha-1} G(1)\right]}{G(1)} \\
& =G(1)\left[1-t^{\alpha-1}\right]\left[1-(1-s)^{\alpha-1}\right] \\
& \geqslant G(1)(\alpha-1)^{2}(1-t) s \\
& \geqslant G(1)(\alpha-1)^{2}(1-t) s t^{\alpha-1}(1-s)^{\alpha-1} .
\end{aligned}
$$

Combining (3.2) and (3.4) with $\mathrm{K}(\mathrm{t}, \mathrm{s})=\mathrm{K}(1-\mathrm{s}, 1-\mathrm{t})$, we have

$$
K(t, s) \leqslant G(1) s(1-s)^{\alpha-1} t^{\alpha-2},
$$

which yields (3).

It follows from (3.3) and (3.8) that (4) holds.

\section{Applications}

In this section, we consider the existence and uniqueness of positive solution for FBVP (1.1).

By Theorem 3.1, we have the following lemma:

Lemma 4.1. The function $\mathrm{K}^{*}(\mathrm{t}, \mathrm{s})=: \mathrm{t}^{2-\alpha} \mathrm{K}(\mathrm{t}, \mathrm{s})$ satisfies:

(1) $\quad K^{*}(t, s)>0, \quad \forall t, s \in(0,1)$;

(2) $\mathrm{K}^{*}(\mathrm{t}, \mathrm{s}) \leqslant \mathrm{G}(1) \mathrm{t}(1-\mathrm{t})(1-\mathrm{s})^{\alpha-2}, \quad \forall \mathrm{t}, \mathrm{s} \in[0,1]$;

(3) $\mathrm{K}^{*}(\mathrm{t}, \mathrm{s}) \leqslant \mathrm{G}(1) \mathrm{s}(1-\mathrm{s})^{\alpha-1}, \quad \forall \mathrm{t}, \mathrm{s} \in[0,1]$;

(4) $\quad K^{*}(t, s) \geqslant M s(1-s)^{\alpha-1} t(1-t), \quad \forall t, s \in[0,1]$.

For convenience, we list here two more assumptions:

$\left(H_{2}\right) f(t, x)=g(t, x, x)$, where $g:(0,1) \times(0,+\infty) \times(0,+\infty) \rightarrow[0,+\infty)$ is continuous, $g(t, x, y)$ is nondecreasing on $x$, nonincreasing on $y$, and there exists $\mu \in(0,1)$, such that

$$
g\left(t, r x, \frac{y}{r}\right) \geqslant r^{\mu} g(t, x, y), \quad \forall x, y>0, r \in(0,1) ;
$$

$\left(\mathrm{H}_{3}\right)$

$$
0<\int_{0}^{1}(1-s)^{\alpha-2} g\left(s,(1-s) s^{\alpha-1},(1-s) s^{\alpha-1}\right) d s<+\infty .
$$

Remark 4.2. Inequality (4.1) is equivalent to

$$
g\left(t, \frac{x}{r}, r y\right) \leqslant r^{-\mu} g(t, x, y), \quad \forall x, y>0, \quad r \in(0,1) .
$$

Remark 4.3. Condition $\left(\mathrm{H}_{2}\right)$ possesses singularity, that is, $f(t, x)$ may be singular at $t=0,1$, and $x=0$. 
Let $E=C[0,1]$ be endowed with the maximum norm $\|u\|=\max _{0 \leqslant t \leqslant 1}|u(t)|$. Define a cone $P$ by

$$
P=\left\{u \in E: \exists l_{u}>0 \text { such that } \frac{M\|u\| t(1-t)}{G(1)} \leqslant u(t) \leqslant l_{u} t(1-t), t \in[0,1]\right\} .
$$

Let

$$
A(u, v)(t)=\int_{0}^{1} K^{*}(t, s) g\left(s, s^{\alpha-2} u(s), s^{\alpha-2} v(s)\right) d s .
$$

Set $Q=P \backslash\{\theta\}$, where $\theta$ is the zero element of $E$. We have the following lemma.

Lemma 4.4. Suppose that $\left(\mathrm{H}_{1}\right)-\left(\mathrm{H}_{3}\right)$ hold. Then $\mathrm{A}: \mathrm{Q} \times \mathrm{Q} \rightarrow \mathrm{Q}$ is a mixed monotone operator.

Proof. The proof is similar to that of [21, Lemma 2.7], so we omit it.

Theorem 4.5. Suppose that $\left(\mathrm{H}_{1}\right)-\left(\mathrm{H}_{3}\right)$ hold. Then the BVP (1.1) has a unique positive solution in $Q$.

Proof. The proof is similar to that of [21, Theorem 3.1], so we omit it.

Similar to [21, Remark 3.1], we have the following results.

Remark 4.6. The unique positive solution $y$ of (1.1) can be approximated by the iterative schemes: for any $w \in Q$, choose $r_{0} \in(0,1)$ small enough such that

$$
r_{0}^{1-\mu} w \leqslant A(w, w) \leqslant r_{0}^{-(1-\mu)} w
$$

Set

$$
u_{0}=r_{0} w, \quad v_{0}=r_{0}^{-1} w,
$$

and $u_{n}=A\left(u_{n-1}, v_{n-1}\right), v_{n}=A\left(v_{n-1}, u_{n-1}\right)(n=1,2, \cdots)$, then $t^{\alpha-2} u_{n} \rightarrow y$.

Example 4.7. Consider the following problem

$$
\left\{\begin{array}{l}
-D_{0+}^{\frac{3}{2}} u(t)+\frac{1}{5} u(t)=f(t, u(t)), \quad 0<t<1 \\
u(0)=0, u(1)=0
\end{array}\right.
$$

where

$$
f(t, x)=t^{-\frac{1}{2}}(1-t)^{-\frac{1}{6}}\left[x^{\frac{1}{4}}+x^{-\frac{1}{4}}\right] .
$$

It is clear that $f(t, x)$ is singular at $t=0,1$, and $x=0$.

Since $\Gamma(\cdot)$ is strictly increasing on $[2,+\infty)$, for any $t \in[0,+\infty)$, we have

$$
\begin{aligned}
g(t) & =-\frac{1}{2 \sqrt{\pi}}+\sum_{k=1}^{+\infty} \frac{t^{k}}{\Gamma\left(\frac{3}{2} k-\frac{1}{2}\right)}=-\frac{1}{2 \sqrt{\pi}}+t+\sum_{k=2}^{+\infty} \frac{t^{k}}{\Gamma\left(\frac{3}{2} k-\frac{1}{2}\right)} \\
& \leqslant-\frac{1}{2 \sqrt{\pi}}+t+\sum_{k=2}^{+\infty} \frac{t^{k}}{\Gamma(k)}=-\frac{1}{2 \sqrt{\pi}}+t\left[1+\sum_{k=1}^{+\infty} \frac{t^{k}}{k !}\right] \\
& =-\frac{1}{2 \sqrt{\pi}}+t e^{t}
\end{aligned}
$$

Since $\frac{1}{2 \sqrt{\pi}} \approx 0.282, \frac{1}{5} e^{\frac{1}{5}} \approx 0.243$, we have $\mathrm{g}\left(\frac{1}{5}\right)<0$. Therefore $\frac{1}{5}<\mathrm{b}^{*}$, which implies that $\left(\mathrm{H}_{1}\right)$ holds.

Denote

$$
g(t, x, y)=t^{-\frac{1}{2}}(1-t)^{-\frac{1}{6}}\left[x^{\frac{1}{4}}+y^{-\frac{1}{4}}\right]
$$

It is easy to check that $\left(\mathrm{H}_{2}\right)$ holds. Through direct calculation, we have

$$
\int_{0}^{1}(1-s)^{-\frac{1}{2}} g\left(s,(1-s) s^{\frac{1}{2}},(1-s) s^{\frac{1}{2}}\right) d s=B\left(\frac{5}{8}, \frac{7}{12}\right)+B\left(\frac{3}{8}, \frac{1}{12}\right)=\frac{\Gamma\left(\frac{5}{8}\right) \Gamma\left(\frac{7}{12}\right)}{\Gamma\left(\frac{29}{24}\right)}+\frac{\Gamma\left(\frac{3}{8}\right) \Gamma\left(\frac{1}{12}\right)}{\Gamma\left(\frac{11}{24}\right)},
$$

which implies $\left(\mathrm{H}_{3}\right)$ holds.

Therefore all the assumptions of Theorem 4.5 are satisfied, which implies that BVP (4.2) has a unique positive solution. 


\section{Acknowledgment}

This work was supported financially by the National Natural Science Foundation of China (11371221), Specialized Research Fund for the Doctoral Program of Higher Education (20133705120003), the Natural Science Foundation of Shandong Province of China (ZR2014AM034, ZR2015AL002) and Project of Shandong Province Higher Educational Science and Technology Program (J14LI08, J15LI16).

\section{References}

[1] R. L. Bagley, P. J. Torvik, On the appearance of the fractional derivative in the behavior of real materials, J. Appl. Mech., 51 (1984), 294-298. 1

[2] Z.-B. Bai, On positive solutions of a nonlocal fractional boundary value problem, Nonlinear Anal., 72 (2010), 916-924. 1

[3] Z.-B. Bai, On solutions of some fractional m-point boundary value problems at resonance, Electron. J. Qual. Theory Differ. Equ., 2010 (2010), 15 pages. 1

[4] Z.-B. Bai, Solvability for a class of fractional m-point boundary value problem at resonance, Comput. Math. Appl., 62 (2011), 1292-1302. 1

[5] Z.-B. Bai, Y.-H. Zhang, Solvability of fractional three-point boundary value problems with nonlinear growth, Appl. Math. Comput., 218 (2011), 1719-1725. 1

[6] J. Čermák, T. Kisela, Stability properties of two-term fractional differential equations, Nonlinear Dynam., 80 (2015), 1673-1684. 1

[7] Y.-J. Cui, Uniqueness of solution for boundary value problems for fractional differential equations, Appl. Math. Lett., 51 (2016), 48-54. 1

[8] V. Daftardar-Gejji, S. Bhalekar, Boundary value problems for multi-term fractional differential equations, J. Math. Anal. Appl., 345 (2008), 754-765. 1

[9] E. F. Elshehawey, E. M. A. Elbarbary, N. A. S. Afifi, M. El-Shahed, On the solution of the endolymph equation using fractional calculus, Appl. Math. Comput., 124 (2001), 337-341. 1

[10] N. J. Ford, J. A. Connolly, Systems-based decomposition schemes for the approximate solution of multi-term fractional differential equations, J. Comput. Appl. Math., 229 (2009), 382-391. 1

[11] J.-Q. Jiang, L.-S. Liu, Existence of solutions for a sequential fractional differential system with coupled boundary conditions, Bound. Value Probl., 2016 (2016), 15 pages. 1

[12] D.-Q. Jiang, C.-J. Yuan, The positive properties of the Green function for Dirichlet-type boundary value problems of nonlinear fractional differential equations and its application, Nonlinear Anal., 72 (2010), 710-719. 1

[13] A. A. Kilbas, H. M. Srivastava, J. J. Trujillo, Theory and applications of fractional differential equations, North-Holland Mathematics Studies, Elsevier Science B.V., Amsterdam, (2006). 2, 2

[14] R. Metzler, J. Klafter, Boundary value problems for fractional diffusion equations, Phys. A., 278 (2000), 107-125. 1

[15] I. Podlubny, Fractional differential equations, An introduction to fractional derivatives, fractional differential equations, to methods of their solution and some of their applications, Mathematics in Science and Engineering, Academic Press, Inc., San Diego, CA, (1999). 2, 2

[16] X.-W. Su, S.-Q. Zhang, Unbounded solutions to a boundary value problem of fractional order on the half-line, Comput. Math. Appl., 61 (2011), 1079-1087. 1

[17] Y.-Q. Wang, L.-S. Liu, Necessary and sufficient condition for the existence of positive solution to singular fractional differential equations, Adv. Difference Equ., 2015 (2015), 14 pages. 1

[18] Y.-Q. Wang, L.-S. Liu, Positive solutions for a class of fractional 3-point boundary value problems at resonance, Adv. Difference Equ., 2017 (2017), 13 pages. 1

[19] Y.-Q. Wang, L.-S. Liu, Y.-H. Wu, Positive solutions for a class of fractional boundary value problem with changing sign nonlinearity, Nonlinear Anal., 74 (2011), 6434-6441. 1

[20] Y.-Q. Wang, L.-S. Liu, Y.-H. Wu, Positive solutions for a nonlocal fractional differential equation, Nonlinear Anal., 74 (2011), 3599-3605. 1

[21] Y.-Q. Wang, L.-S. Liu, Y.-H. Wu, Existence and uniqueness of a positive solution to singular fractional differential equations, Bound. Value Probl., 2012 (2012), 12 pages. 1, 4, 4

[22] Y.-Q. Wang, L.-S. Liu, Y.-H. Wu, Positive solutions for a fractional boundary value problem with changing sign nonlinearity, Abstr. Appl. Anal., 2012 (2012), 12 pages. 1

[23] Y. Wang, L.-S. Liu, X.-U. Zhang, Y.-H. Wu, Positive solutions of an abstract fractional semipositone differential system model for bioprocesses of HIV infection, Appl. Math. Comput., 258 (2015), 312-324. 1

[24] X.-J. Xu, X.-L. Fei, The positive properties of Green's function for three point boundary value problems of nonlinear fractional differential equations and its applications, Commun. Nonlinear Sci. Numer. Simul., 17 (2012), 1555-1565. 1

[25] X.-J. Yang, Fractional derivatives of constant and variable orders applied to anomalous relaxation models in heat-transfer problems, Therm. Sci., 2017 (2017), 12 pages. 1

[26] X.-J. Yang, D. Baleanu, H. M. Srivastava, Local fractional integral transforms and their applications, Elsevier/Academic Press, Amsterdam, (2016). 
[27] X.-J. Yang, F. Gao, J. A. Tenreiro Machado, D. Baleanu, A new fractional derivative involving the normalized sinc function without singular kernel, ArXiv, 2017 (2017), 11 pages.

[28] X.-J. Yang, H. M. Srivastava, J. A. Tenreiro Machado, A new fractional derivative without singular kernel: Application to the modelling of the steady heat flow, Therm. Sci., 20 (2016), 753-756.

[29] X.-J. Yang, J. A. Tenreiro Machado, A new fractional operator of variable order: Application in the description of anomalous diffusion, Phys. A, (2017), (In press).

[30] X.-J. Yang, Z.-Z. Zhang, J. A. Tenreiro Machado, D. Baleanu, On local factional operators view of computational complexity: Diffusion and relaxation defined on cantor sets, Therm. Sci., 20 (2016), S755-S767. 1

[31] C.-B. Zhai, L. Xu, Properties of positive solutions to a class of four-point boundary value problem of Caputo fractional differential equations with a parameter, Commun. Nonlinear Sci. Numer. Simul., 19 (2014), 2820-2827. 1

[32] Y.-H. Zhang, Z.-B. Bai, T.-T. Feng, Existence results for a coupled system of nonlinear fractional three-point boundary value problems at resonance, Comput. Math. Appl., 61 (2011), 1032-1047. 1

[33] X.-U. Zhang, L.-S. Liu, B. Wiwatanapataphee, Y.-H. Wu, The eigenvalue for a class of singular p-Laplacian fractional differential equations involving the Riemann-Stieltjes integral boundary condition, Appl. Math. Comput., 235 (2014), 412-422. 1

[34] X.-U. Zhang, L.-S. Liu, Y.-H. Wu, The eigenvalue problem for a singular higher order fractional differential equation involving fractional derivatives, Appl. Math. Comput., 218 (2012), 8526-8536. 1

[35] X.-U. Zhang, L.-S. Liu, Y.-H. Wu, The uniqueness of positive solution for a fractional order model of turbulent flow in a porous medium, Appl. Math. Lett., 27 (2014), 26-33. 1 